\title{
EVS24
}

Stavanger, Norway, May 13-16, 2009

\section{Comparison of packaging hydraulic and electric components in a HEV power line}

\author{
Panu Sainio ${ }^{1}$, Matti Heiska ${ }^{2}$ Teemu Lehmuspelto ${ }^{3}$, Jussi Suomela ${ }^{2}$ \\ ${ }^{I}$ Helsinki University of Technology TKK, Department of Engineering Design and Production, Vehicle Engineering, \\ PL 4300,02015 TKK, Finland, panu.sainio@tkk.fi \\ ${ }^{2}$ Helsinki University of Technology TKK, Department of Automation and Systems Technology, Generic Intelligent \\ Machines \\ ${ }^{3}$ Helsinki University of Technology TKK Department of Engineering Design and Production, Vehicle Engineering
}

\begin{abstract}
Scientific papers usually do not present 'lessons learnt' results, what comes to engineering aspects in vehicle design. A lot of valuable information is collected during research and prototyping projects, but this is not shared in public often enough. This paper is a general discussion about the engineering matters related to the implementation of hybrid systems in mobile work machines. The target of this paper is to underline the importance of paying attention of these matters already in very beginning of the research and development.
\end{abstract}

The discussed aspects are related to the energy transmission, packaging of components, vehicle assembly and service. The energy transmission section compares benefits and drawbacks of hydraulic and electric transmission implementations. Packaging of components presents some issues to pay attention to when accommodating components into vehicle. Service aspect highlights the benefits and drawbacks of different solutions in maintenance operations.

The discussion is limited to explain only differences and analogies between hydraulic and electric components. The cost factors can be estimated only by the reader, because they are very case sensitive. The key factor is number of machines made in serial production. In case of passenger cars the cost structure is very different compared to small series mobile work machines.

Keywords: hydraulic pipe, hydraulic tube, electric wire, electric cable, power cable, connector, assembly, service

\section{Introduction}

General discussion and remarks about the engineering matters are often ignored in result focused scientific papers. Engineering aspects may be considered to be as well-known facts or they are too general for scope of the papers. These general engineering remarks are valuable when new technologies are adapted to new applications. It helps to get the common acceptance for new technologies inside the company, among suppliers and development partners, and most importantly within market and customers. Any kind of documented information is also helpful for young engineers and engineering companies, or engineers with different technical backgrounds to understand the benefits and drawbacks of different technologies. 
This paper points out some findings made during some hybridization projects of heavy vehicles and mobile work machines. These machines are usually dominated by hydraulics, whereas high power electrical systems are relatively new. For hybridization projects there is a demand in the future to understand constrains and advantages of these two different ways to move energy inside mobile work machines.

The items discussed here will be related to the power transmission, packaging, assembly, and service when using hydraulic or electric systems. In this paper there are only general remarks of differences and analogies between hydraulic and electric components. The cost factors cannot be discussed here. They depend very much of the volume of production. Readers can find out how much is too much by calculating the payback period if all lifetime costs are considered.

Often engineers have strong opinions which of these two technologies are the best, based on their background on mechanical or electrical engineering. This paper tries to give an objective opinion of both of them. It is also important to point out, that the best solution combines the best characteristics of these technologies and support each other in their weaknesses.

\section{Power transmission}

\subsection{General}

Today diesel engine is the most common energy source in mobile working machines. What comes to power transmission from engine drive shaft to systems, mechanical and hydraulic links are used. Electricity is a new option for power transmission in hybrid vehicles and even mandatory in fuel cell powered machines.

Mechanical power line is very common, but not very simple or cost effective solution for power transmission. With mechanical transmission there is often need for several different gear ratios including some steps. Mechanical power transmission is used in vehicle drive lines. However, in some cases, e.g. with bucket movements in wheeled loader, the mechanical power transmission is even impossible to implement in reasonable way.

Hydraulics, as a whole has several great benefits and features what have made it to dominate as transmission path for energy in mobile work machines. The flexibility of hydraulics is much better than in mechanical power transmission. Hydraulics can be used to change the gear ratio continuously with variable displacement pumps and motors. It also has a great power density, which make it beneficial to use hydraulics in narrow places where high power is needed, like with bucket.

Electricity is the most flexible way to deliver power between systems. The drawback is the lack of components for heavy machinery. Electric motors usually have lower torque but higher speed compared to hydraulic motors. That is why more gear ratio is needed between electrical actuator and system if we compare it to hydraulics. In many cases, the electric motor with a gearbox is often too big to place it near to the actuator. The beneficial use of electrics would need a total reconstruction of the machine and new kind of electrical actuators.

\subsection{Characteristics of hydraulic and electrical transmission paths}

The scope of this chapter is to compare hydraulic and electrical transmission paths. It is mainly remarks for comparison of hydraulic hose and electric cable. These remarks are somewhat application sensitive and can be utilized when having discussion cost structure and engineering principles of hybrid power lines in moving work machines.

Hydraulic hose is a high tech product despite of its "round and black" type of appearance. Manufacturing, selecting and making the proper, cost effective assembly demands much more than is visible to outside. The structure of hydraulic hose is presented in Fig.1.

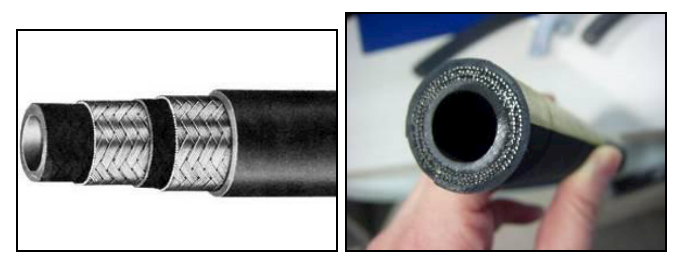

Figure 1: Structure of typical hydraulic hose is presented in the picture. It is flexible rubber hose with different types of reinforcements typically made from metals. Hydraulic tube or pipe is typically a firm metal tube

The demand for high quality manufacturing is the case also with electric cables. Signal and control 
cables are somewhat different, but having a number of wires and very complicated connectors they are worth of own review. The structure of shielded cable is presented in Fig.2.

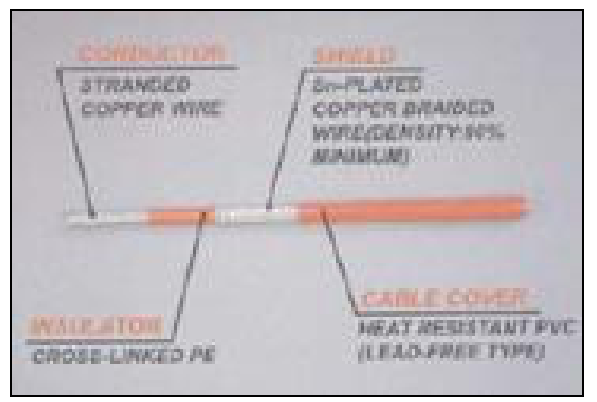

Figure 2: Structure of typical electric cable for HEV use [5]

Hose in Fig.1. and cable in Fig.2. has similar structure, but for different reasons. The core of the cable and hose is for power transmission. The outer metal layers have different purposes. However, the protection against mechanical wear and chemical stress are common characteristics for both.

In hydraulic hose the inner radius is dependent of flow. Typical inner diameter for $100 \mathrm{~kW}$ power is $19-25 \mathrm{~mm}$. Outer diameter of hose depends on the pressure rating, because more reinforcement layers and higher wall thickness is needed. The outer diameter is $33-40 \mathrm{~mm}$ for 1 " hoses with pressure ratings required in mobile work machines.

Cable in Fig.2. can be used in same power class as the hydraulic hose mentioned earlier. The core in $35 \mathrm{~mm}^{2}$ multithread cable has diameter of 10 $\mathrm{mm}$. The outer diameter depends on insulation requirement. The metal shield depends on the requirement for electromagnetic compatibility. In this example the cable has outer diameter of 15 $\mathrm{mm}$ and it is accepted in vehicle use.

The example above shows, that the need for space is five times more with hydraulic hose than with cable. Actually this is much more, because hydraulic hose is stiffer and the allowed minimum bending radius of hydraulic hose is much higher.

Hydraulic hoses and electric cables can be replaced by hydraulic tubes and busbars, if the space is more limited or if there is a need for more rigid assembly. Hydraulic tube has the same requirements for inner radius than tubes. The outer diameter can be few millimeters less. Tighter corners can be made with tubes, but you have to remember the effect on flow quality of hydraulic fluid and pressure. Too tight corners and increased number of connectors and fittings causes power losses. They can have significant effect on energy efficiency at high power.

Busbars are used in rigid electric system installation. Basically they can be any size and shape. Usually the cross section is rectangular, not round like with cables. Tight corners are not a problem in case of power losses. Disadvantage is that shielding against EMC is difficult. High frequencies affect electrochemical actions on surface of busbars, which creates a need for proper coating to avoid oxidation and poor contacts between busbars.

Using busbars is common solution inside power electronic devices. This technique offers multiple benefits for assembly and dissipations. In future perhaps busbars might be used also between components and they could be fixed in vehicle frame. There can be seen new application with busbars made of thin sheets with insulation (see Fig.3.). These might offer us cost benefits what comes to connecting, guidance and continuous current-carrying capacity. [2]
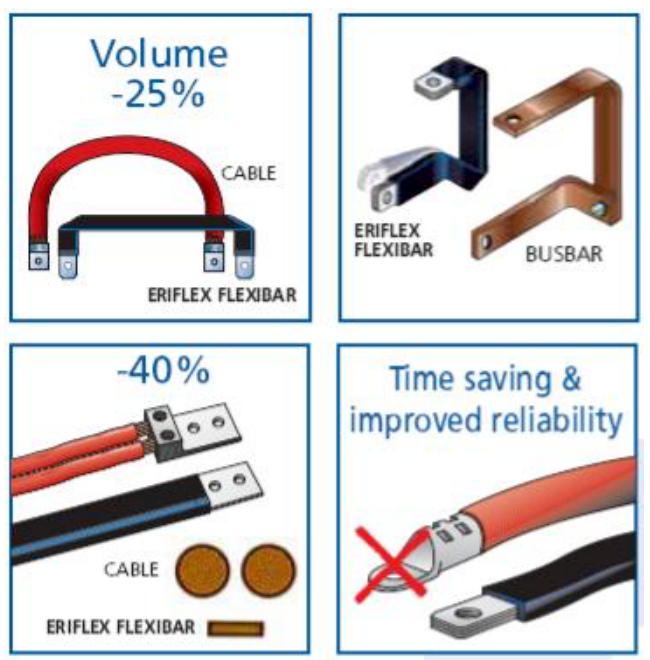

Figure 3: The summarization of the advantages claimed for modern insulated busbars compared to the classical electric cabling. [2] 


\subsection{Connections}

There are much different type of connections for hydraulic hoses and tubes, as well as electric cables and busbars. We all who have been struggling with crimping, pressing or even soldering connectors to cables have seen how difficult it is. The very same or even worse situation is with hydraulic hoses. There is demand for special tooling and there are numerous differences between fittings and sealings. Fig.4. is really only a glimpse from that world.

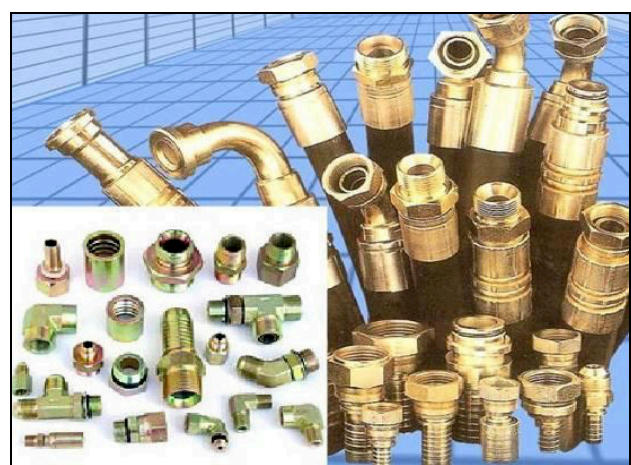

Figure 4: Fitting for hydraulic hoses

There is said a very rough estimation the third of the turnover of hydraulic systems industry would be related to hoses, pipes, fitting, filtering and other that kind of stuff. This rule of thumb estimation could perhaps be used as a reference when comparing to the costs of cabling, connectors and fuses.

The simplest way with electric cabling is bolt connection. This can be made with different types of cable lugs. Flexible busbar in Fig.3. allows making bolt connections by punching directly through the laminates. Since there are no lugs to purchase, fewer parts are required and installation is simpler and faster. Compared to cables tools to make this kind of solutions are simple enough for research, prototyping and even in serial production. However, this kind of noninsulated connection can only be used inside a housing or additional protection against contact and electric shock must be implemented. Fig.5. shows an example of safe and EMC shielded cable connector for high voltage systems in HEV.

Easy bolt connections are not possible in hydraulics. The closest to do that is probably a banjo bolt or flange mounting, but compared to bolt connection for busbars, they are much more impractical. The benefit of connectors of hydraulic tubes and hoses is that they don't need extra protection. Fittings for hydraulics are always secured for rated pressure if installed properly. HEV.

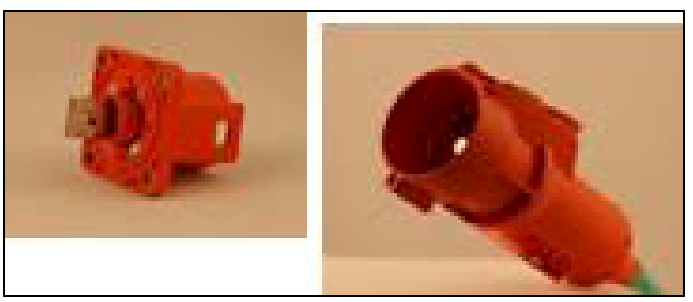

Figure 5: Connector for high voltage system [5]

Both hydraulic hose and electric cable connectors have larger diameter that the hose or cable itself. This means that there is need for large lead ins. Of course this offers good possibility for proper bushing for leads in but has to be considered for assembly. Especially when it comes to manufactured wire harness there is good possibility to have demand large cross section in the cable routing. For service a large connector with angle at the end of stiff hose is for sure a challenge.

\section{Manufacturing and assembly}

\subsection{General}

The engineering task of packaging and assembly components in the vehicle is a complex matter. It includes a lot of tacit knowledge inside organization, and it is rarely documented. Often this tacit knowledge is gathered by experience and co-operation with service people. The aspects in this chapter are heat transfer and general manufacturing and assembly issues.

\subsection{Packaging and heat transfer}

Extensive heat production due to bad efficiency and constant demand for cooling has always been the challenge in vehicles and mobile work machines. With combustion engine and hydraulics a certain level of temperature is wanted for proper operation. There are remarks to warm engines before using full power. The viscosity of hydraulic fluid, which is highly dependent of fluid temperature, has a great effect on system behavior. The hydraulic fluid has certain operation temperature about 50-80 degrees of Celsius. With electric components typically all heat is unwanted and the overall accepted temperature level is 
lower. Usually it is under $65{ }^{\circ} \mathrm{C}$ in power electronic devices of today. These different thermal levels have to be taken into account while engineering packaging.

The hydraulic oil has very important function of cooling besides energy transferring. The target temperature for hydraulic oil is relatively cool in work machine operated by combustion engine at outdoors in summer. But when comparing this temperature of demands rising from electronics, battery or capacitor, it is much more difficult. In conventional mobile work machine the surface temperature of hydraulic pipes and tubes is not a major worry. They also have significant role of cooling down the hydraulic oil. Insulating them will lead us to increscent cooling capacity to some other location. For protecting single components insulating or routing hydraulic line other way around are good solutions.

When making hybridization of mobile work machine, one has to remember the heat flow from hydraulic lines. The advantage of electric power cables is that they are targeted not to rise in temperature significantly due to current.

Hydraulic system also generates heat during idle. Even if the bucket is not moving but the combustion engine is running and circulating the oil, there is heat generation from few up to dozens of kilowatts. Fig.6. presents this fundamental difference between hydraulic and electric energy transfer paths. The hydraulic systems start to heat up immediately after start due to oil circulation, electric until when working. Electric cables as transfer paths do not produce heat while idle and electric system as a whole has very low heat generation during idle. This might be used as an advantage while engineering cooling system for hybrid work machine. Especially for those components, which are used seldom during the working cycle, there might be possibilities to survive with limited cooling capacity. Here we have to remember that there are major differences of working cycle of different working machines and their functions. Specifying and identification of the working cycle of a certain working machine is one of the first steps in hybridization feasibility study.

For hydraulic fluid and cooling of it advantage is that it can be piped to separate cooler. If there is excessive heat production inside electric devices, typically a separate water cooling circuit is needed. If in worst case, there is from some reason excessive heat production inside cabling, there is not much to be done. Of course type and sizing of the cable as well as the mounting and the routing of the cables may offer some instruments to control the temperature.

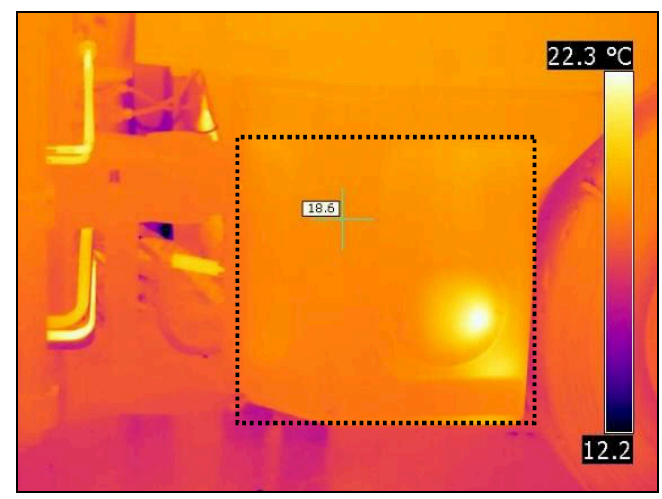

Figure 6: Example of an infrared picture taken from the hydraulic oil tank only 2 minutes after start up the loader. The pipes on the left side are for bucket hydraulics and on the left marked with dashed line is a 150 litres hydraulic tank.

It is also worth of mention, that development of high power semiconductors is focused on more heat sensitive components. The temperature of power electronic devices could be increased by 10$20{ }^{\circ} \mathrm{C}$ in the near future with silicon carbide based semiconductors.

\subsection{Vehicle installations}

For costs, wear and ease of guidance in hydraulics, there are used pipes for longer distances, and hoses with minimum length in locations, where is demand to allow movements. Hydraulic hoses are subjects of wear when mounted in moving component by external abrasion and there is also possibility for internal erosion.

Hose routing is a challenge, as well it is a major matter with electric cables. Replacement hoses must be restrained, protected, or guided typically using clamps to minimize excessive flexing and whipping. Flat metal armor and wire spring guards, or a special sheath made of abrasion, temperature, or chemical resistant material will help protect a hose. Electric cables have similar needs but with major difference not having tendency to whipping due to change of power flow. Hydraulic hoses vibrate and change their stiffness when power flow is varied. 
Also when determining correct hoses length, consideration must be given for length changes under pressure (being as a rule of thumb about $5 \%$ ), machine vibration and motion, and hose assembly routing. A special remark for hydraulics is that high and low pressure lines should not be clamped together. In electric cables there are no change of the length and stiffness. However in electric cables one should consider when clamping together cables with different types of signals and levels of power. The engineering of wire harness in terms of conduction of heat and EMC-matters can be a challenge.

Fig.7 presents situation of center pivot in articulated frame steered wheel loader. There is need to transfer energy by hydraulic hoses over that joint and with very limited space. This makes hydraulic hoses to contact each other. Also there are hoses for high and low pressure, and for different circulation. This makes hoses to move at each other due to pressure fluctuation.

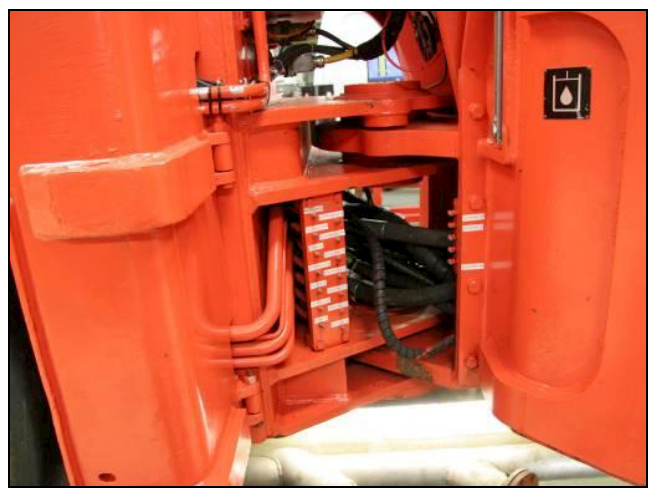

Figure 7: Hydraulic hoses in wheel loader with articulated steering (frame steering). This typically challenge both for assembly and external wear of the hoses.

Center pivot is a challenging point also for electric cabling. But as learnt earlier, electric cables are more flexible and don't need so much space. They do not change their stiffness as a function of energy either. The electric cables are also subject to external wear and we have to remark there is limited experience of them in connection between moving parts. This may force us to protect soft cabling by canal or such. Presence of oil and other chemicals form an other threat to cabling. However the same situation is with hoses.
Fig.8. shows the flexibility of electric cabling in vehicle installations. It is a picture of electric vehicle prototype in very early phase of functional tests. Compare them in your mind to hydraulic hoses and tubes. There are differences of sizing, bending radius, heat generation and demand for support. A lot of extra work has been neglected in designing the power transmission paths and installations, because of the freedom what comes to lay-out and cable routing. Although this is just a case from prototyping phase, the same freedom remains in production phase.

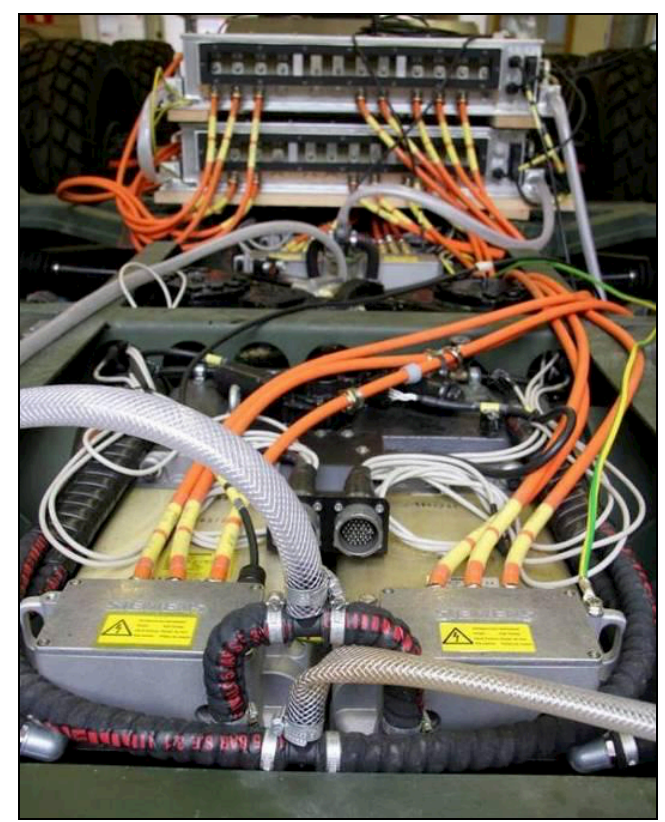

Figure 8: An example of packing two Siemens electric motors during first bench testing. Remark there is power cables, cooling pipes and control cables. [3]

Typically in both of our main cases, electric cabling and hydraulic hoses and tubes are forgotten in press material or even during making the first engineering concepts. Cabling and hoses do demand volume inside of the vehicle or working machine. In Fig 9 and Fig. 10 there are two typical presentations. It is natural, understandable and acceptable the new systems are presented to the audience only by pointing out the main components and their main relations. However the reader has to keep in mind when making the estimation of the feasibility of the new systems, that most of the component has to be connected by several means. There is very often need for at least two hoses and some signal cabling. 
In case of power electronic components cabling might be more assembly and service friendly by good reasons. When using shielded cables due to EMC-matters we achieve at the same time only two connection for one component. Having power phases inside same cable and all signal/controls in the other cable the assembly easies up and we ensure to have better EMC performance.

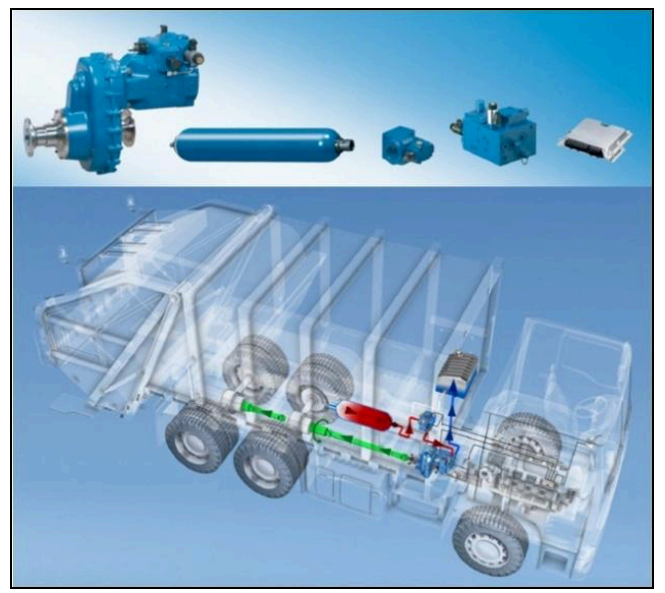

Figure 9: Components of the hydraulic hybrid Hydrostatic Regenerative Braking System. This is very common way to present component in marketing material, without pipes and cables although they will be a challenge during application engineering. [1]

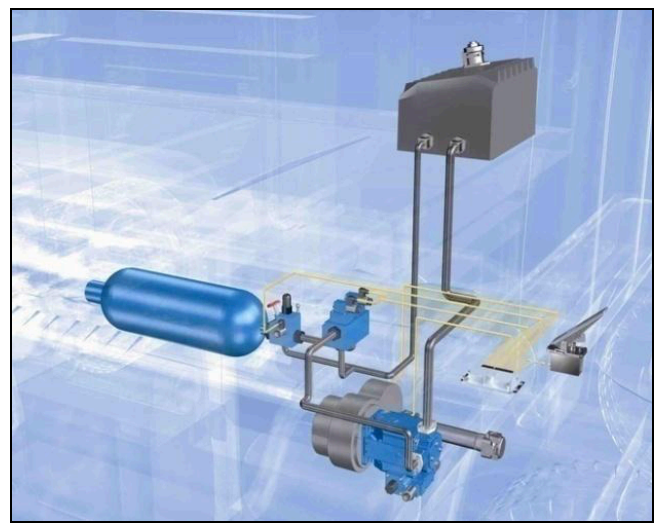

Figure 10. Here is much more realistic example of marketing picture including some tubing and wiring. Remark even presentation of hydraulic fluid tank in somewhat reasonable size. [1]

\subsection{Assembly work}

The engineering items related to assembly and the targeted service life of hydraulic hoses and tubes are worth of short discussion. There is direct analogy from many of these items to electric cabling. However, there are some items that are not present with electric power cables. These can be considering as benefits to hybrid electric systems. In discussion they have to be often underlined because in the mobile work machine industry all these aspects related to hydraulics are accepted as a part of everyday business and cost structure.

Hoses, couplings, assembly equipment, and crimping tolerances vary from one manufacturer to another, and sometimes they are not interchangeable. The main worry is to remember that the thread, seal or flange end of a coupling must be matched properly to other component to achieve a leak proof connection and safe. This problematic is similar to connectors in electronics. When making power cable connections with bolt-based joint some of this is surmounted.

There is also more difficult to design rigid hydraulic lines in to different sub modules so, that they could be fitted together when installing them together. In this case, only option is to use a piece of hose between those modules to connect the power line together. This is much easier and accurate with electric busbars and cable connections. You could even imagine fixing a rigid electric bus in vehicle frame, and having a counterpart in sub module to fit to that in tolerance of few millimeters.

When making the first assemble, and sometimes also during service, there is need in hydraulics to flush the system to prevent impurities to remain the system. In electric cable there is sometimes need to measure leakage current but no need to flush the system.

\subsection{Safety aspects}

Generally high voltage electric systems are considered to be more dangerous than hydraulics. For electric cables, if there comes a crack or a cut there is a danger for short circuit. For this there are possibilities to be prepared by proper fuses and current leak detectors. It is also mandatory to have a protection against contact to avoid electric shocks.

With hydraulic hoses there is danger for high pressure fluid to be sprayed or fumed out of the leakage point. High pressure spray may be lethal or at least leads to permanent tissue damage. Fume is dangerous for its risk of fire. Both of them make environmental risk of having hydraulic fluid (even 
tens or hundreds of liters) around the working site. Typically for customer it is easy to ask and to be afraid of the danger of electric cable to be cut. But at the same time the same customer accepts and can live with the danger of hose failures and some regular, external leaks of hydraulic systems.

Temperature range of the hoses is a matter of choice with remark that hydraulic hoses must tolerate both the external ambient and internal fluid temperatures. Fluid compatibility has to be considered. Many hoses are not compatible with all of the common fluids that include petroleumbased, phosphate esters, water-based and diesters. Here we have only limited analogy to electric cables.

Standards and references for combustion and electric vehicles are today under intensive development. Inside traditional electrical industry there has been variety of norm, guide lines and standards to be followed. Often there is assumption of being stationary assembly or in case of moving vehicle low voltage (like forklifts with 72 volts etc).

Electrical safety and even in some cases security has to integrated in to the life span of the vehicle or working machine. [6] This means we have to consider safety as an item covering engineering, testing, and assembly maintenance and even recycling phases of the product.

\section{Service and repair}

The service costs are often calculated carefully in case of mobile work machines. They are handled as investments and factor of production. However, if and when there becomes demand for repair, the effective process for trouble shooting is needed.

In operations model, where work machine is owned the same owner as manufacture (case of group of companies or affiliated company etc.) and it is leased including service, the service cost become more and more important. Practically when there is clause of operational availability of allowed down time, the role of trouble shooting may becomes very valuable. Via this kind of items the higher price of hybrid systems may become feasible as part of total cost structure. The saving of the energy is only a one part. Especially this is valuable if the tendency of leasing work machine even based on actual performance becomes more common.

Service can be done either by beforehand planned model or because of failure remarked. Often actions do to failure are referred also by term of repair. In future however, there can be predicted demand for service when really needed, so service in demand. There is assumption of having usage profile and operating conditions predictable, if service interval is based on working hours. For example in hydraulics there is a good possibility to indicate service for filtering via pressure difference over the filter, which indicates fulfillment of the filter.

For the hoses there is only a visual inspection. That may be difficult because hoses are clustered and they are inside the machine. Of course leakage is an effective marker itself, that there is a problem somewhere. However, it can be difficult see in what component the leakage actually is. Oil under the machine can be detected at least on hard soil, but finding the exact section may be difficult. It is also possible, that leakage is present only at high temp and full power.

In case of electric cabling there should be possibilities to measure cable sections separately, and define in which section or location the problem is. Also self diagnostics could be exploited and taken into account already during engineering phase of the system [4].

What comes to assembly and service demands for easy connection and possibility to open and reconnect it to the hydraulics can fulfil them mostly. However when removing electric cable there is no fluid running to floor and into the machine as well as there is no major danger for impurities entering to the system. This is clear bonus for electric cables. Especially this is important for mobile work machines, because many service tasks are done where the machine happens to be at the time of breakdown.

One difference claimed between cables and hoses is case of great damages inside of the system. It is sometimes believed in case of hydraulic pump, motor or other metallic component major damage that the machine will never be the same again. There is obvious need to flush the system to get metal particles away. But before noticing the damage or excessive wear the particles may easily spread to the whole system. And after the repairs and flushing, despite of several levels of filtering, 
there can be some parts of metal left in the system. In case of cables the damage is typically isolated to certain components by the assumption the over voltage protection is done properly.

Manufacturing spare parts for the vehicles of tomorrow is one item should be considered now. In case of hydraulic hoses and electric cables there is need for supply and availability of selected connectors. This should not be a problem, if standards are followed. Especially with cables done with bolt joints and lead-ins this should not be a problem. For hydraulics there may be differences of threads and sizing between continents and market areas what may cause difficulties to customers later on.

\section{Conclusions}

Items discussed here may be not the first points in presentation material of novel hybrid power line solutions for mobile work machines. However, these aspects and observations might be useful when new technology enters to production phase or even before that when having challenge of price setting, reliability estimation, payback time calculation to achieve customer acceptance. Saving energy is one obvious driving force in development of hybrid system, but it has to be noticed that also there has to be payback time, reliability and good cost structure before any system can enter to production and markets.

Electric power lines can bring more flexibility and cost saving in manufacturing and maintenance of mobile work machines. It is clear that it is the most reasonable way to transfer power between systems. However, hydraulic systems and components are still present in mobile work machines because of the very great power density of hydraulics.

When combining the best sides of electrics and hydraulics, it makes sense to conclude to series hybrid construction in mobile work machines. The energy system with gen-set and electrical energy buffers with sophisticated control system improve fuel efficiency. Electrical power lines makes and serial hybrid structure can give us more freedom in engineering. Electric driven actuators benefits of hydraulic gear ratio, and can be used close to the actuator.

\section{Acknowledgments}

Our gratitude is to all those of our friends, colleagues, partners and customers who have been explained details, discussed solutions and have been giving their constructive criticism about engineering items we have phased during several projects in the wide field of work machinery.

This paper is part of an ongoing project $H Y B L A B$ funded by the Multidisciplinary Institute of Digitalization and Energy (MIDE) of Helsinki University of Technology TKK.

\section{References}

[1] www.bosch.com, referred 2.4.2009

[2] www.erico.com, referred 10.4.2009

[3] Jussi Suomela, Panu Sainio, Peter Jakubik, Antti Leivo, Markku Degerholm, Teemu Lehmuspelto, Control system development for an 8-wheel off-road HEV by using $\mathrm{V}$ cycle, Poster at EVS-22, October 23-28, Yokohama, Japan 2006

[4] Matti Juhala, Panu Sainio, Antti Leivo, Development environment for heavy vehicle hybrid systems, 8th International Workshop on Research and Education in Mechatronics 2007, June 2007, Tallinn, Estonia

[5] Yazaki, www.yazaki-na.com, referred 14.4.2009

[6] Jacobs, U., Rau, M., Anforderungen an Hybridsysteme in Bezug auf Funktionale und Elektrische Sicherheit aus normativer Sicht (Requirements to hybrid systems with regard to functional and electrical safety in view of standards) TÜV SÜD Automotive $\mathrm{GmbH}$, VDI Berichte Nr. 2000, 2007 509-526 


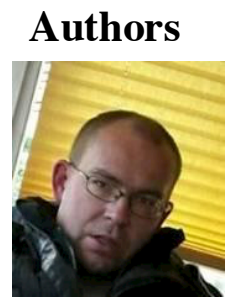

Panu Sainio, Lic.Tech. Chief Engineer, Department of Machine Design and Production, research group for Vehicle Engineering, panu.sainio@tkk.fi

$\mathrm{He}$ has been working as

Laboratory Manager and later as Chief Engineer since 1998 participating a number of national and international projects of vehicle engineering and testing. His research interests are tyre-road interaction, non-visual information for driver's and heavy hybrid vehicles.

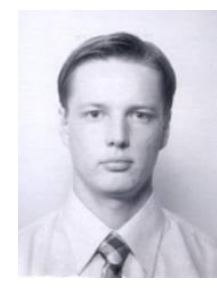

Matti Heiska received his M.Sc. degree in Vehicle Engineering from Helsinki University of Technology (TKK), Finland, in 2002.

matti.heiska@tkk.fi

During 2002-2008 he worked in

Patria Land \& Armament as a R\&D

Engineer. Since 2008 he has been working as a Research Scientist in Department of Automation and Systems Technology in TKK. His main research interests are in the field of vehicle control systems and hybrid electric vehicle technologies

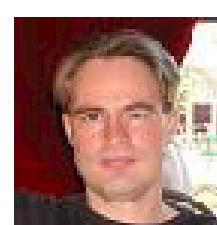

Teemu Lehmuspelto, M.Sc. Research Scientist, Department of Machine Design and Production, research group for Vehicle Engineering .

teemu.lehmuspelto@tkk.fi

He has been working as a R\&D Engineer 2001-2008 in Patria Vehicles Oy and as a Research Scientist since 2008 in Helsinki University of Technology. His main research interests are vehicle control technologies and hybrid electric vehicle technologies.

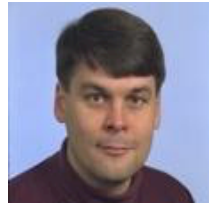

Jussi Suomela is senior research scientist and project manager in Department of Automation and Systems Technology in Helsinki University of Technology TKK since 1992. His main research areas are hybrid electric vehicles and field and service robotics. He received his doctoral degree from TKK in 2004. jussi.suomela@tkk.fi 